\title{
Small bowel Ascaris infestation: a diagnostic challenge
}

This article was published in the following Dove Press journal:

International Journal of General Medicine

26 April 2016

Number of times this article has been viewed

\section{Muhammad Waqas Khan Sanniya Khan Ghauri \\ Section of Medicine, Department of Emergency Medicine, WHO Collaborating Centre for Emergency Medicine and Trauma Care, Aga Khan University Hospital, Karachi, Pakistan}

Correspondence: Muhammad Waqas Khan

Section of Medicine, Department of Emergency Medicine, WHO Collaborating Centre for Emergency Medicine and Trauma Care, Aga Khan University Hospital, Stadium Road, 74800, Karachi, Pakistan
Email mwkhan6I@gmail.com

Abstract: Ascariasis is a common infestation in developing countries where there is poor hygiene. A majority of the cases are asymptomatic, with a few cases presenting with mild abdominal pain and nutritional deficiencies in the long term. Here we present a case of a young boy who presented as a diagnostic dilemma, with signs of acute intestinal obstruction without any supporting radiological evidence. A barium study revealed the presence of low-burden Ascaris infestation that was managed medically.

Keywords: partial intestinal obstruction, gastro-intestinal helminthic infection, pediatric

\section{Introduction}

Ascariasis is a common infestation in developing countries where there is poor hygiene. Most of the cases are asymptomatic with the exception of a few that may present with mild abdominal pain and nutritional deficiencies. Intestinal obstruction is an acute presentation that has been observed in a number of cases where there is an increased worm burden that may partially or completely block the intestinal lumen. ${ }^{1,2}$ We present a case that proved difficult to diagnose as the child's symptoms of subacute intestinal obstruction did not pair up with the radiological results, forcing us to take unusual measures for diagnosis.

\section{Case report}

A 3-year-4-month-old child presented to the emergency department with the complaints of greenish vomiting and abdominal pain for 3 days. His vomiting bouts were continuous with a frequency of five to ten episodes a day - each around half a cup. Vomitus contained greenish fluid with no food particles, blood, or mucus. He had been passing stool until his presentation at the hospital. He had a history of mild constipation for 1 year, for which he was being managed with a high-fiber diet.

On examination, the child was thin, irritable, and moderately dehydrated. Though mildly tachycardic (heart rate of 125 beats per minute), his respiratory rate, temperature, and blood pressure were within reference range. Abdomen was soft and nontender with sluggish gut sounds. Laboratory investigations showed an increased leukocyte count $\left(14.5 \times 10^{9}\right.$ cells/L; neutrophils, $79.9 \%$; lymphocytes, $10.9 \%$; eosinophils, $2.2 \%$; basophils, $0.1 \%$ ) without any abnormalities in serum concentrations of electrolytes, amylase, and lipase.

Supine X-ray of the abdomen was performed suspecting intestinal obstruction, which showed no abnormalities (Figure 1). Computed tomography (CT) of the 


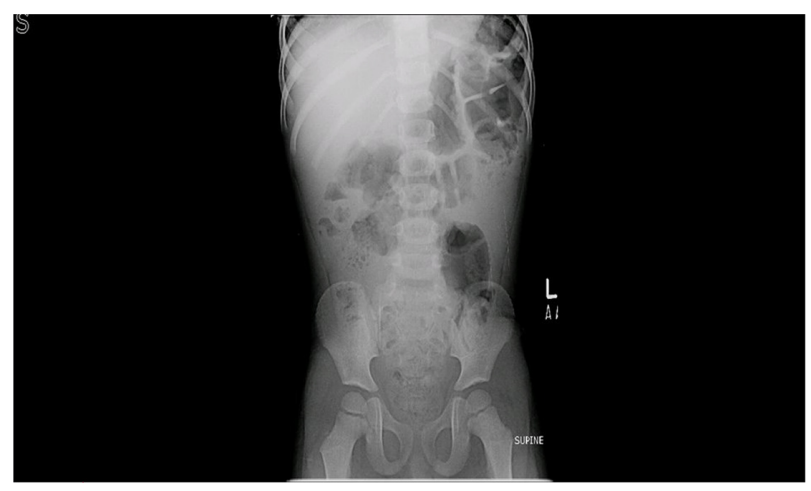

Figure I Abdominal X-ray.

abdominal region was done to rule out any anatomical or positional abnormalities of the intestine, with suspicion of a partial obstruction in the small intestine. The CT revealed no abnormalities either. The child was withheld oral food and fluid (nil per os), and nasogastric tube was kept on low volume suction. Barium meal and follow through were hence scheduled, which revealed growth of intestinal worms in the upper intestine at various sections, which were found to be the cause of partial intestinal obstruction, as shown in Figure 2. The number of worms found was quite low, leading to the inability of reaching a definitive diagnosis earlier. Upon resolution of the patients vomiting bouts and the development of healthy bowel sounds, he was initially started on a liquid diet and his diet was progressed further. Albendazole was given as an antihelminthic and the patient was discharged. Follow-up after 6 days showed complete resolution of symptoms with confirmation that the worms had been passed during defecation. This study was conducted

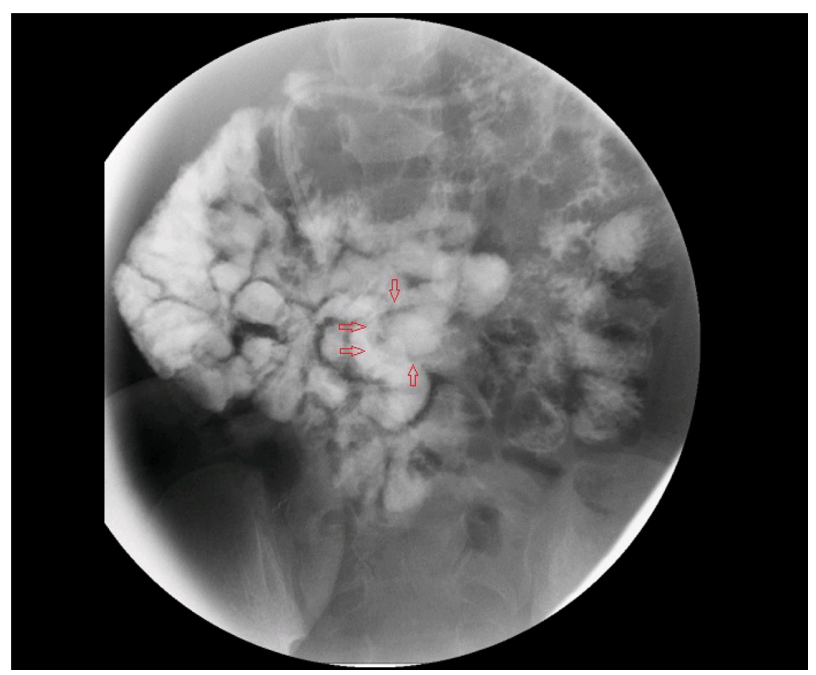

Figure 2 Barium image.

Note: Arrows indicate the outline of the worm. with the written informed consent of the patients guardian, and was approved by the ethical review board of the Aga Khan University Hospital.

\section{Discussion}

Intestinal nematode infection is one of the leading five causes of intestinal obstruction in a third of the world's population. Ascaris lumbricoides (AL) is the most common form of helminthic infection responsible for several medical problems, especially in the developing countries. ${ }^{2}$ Though a vast majority of cases are asymptomatic, infected patients may present with a potentially severe variety of pulmonary and gastrointestinal disturbances. ${ }^{1-3}$ Ascaris helminthic infection is predominantly found in areas of poor sanitation and is associated with malnutrition, anemia (iron deficiency), and impairments of cognition and growth. Intestinal obstruction is one of the rare and more lethal complications.

AL usually affects the pediatric age group patients residing in low socioeconomic areas with malnutrition and immune deficiencies. The worms reside near the ileocecal valve without causing serious symptoms, unless the environment becomes too hostile for their survival, which may result in their migration to a more tolerable region in the intestinal tract. AL migration may cause serious complications such as pulmonary ascariasis, pancreatitis, liver abscesses, cholecystitis, volvuli, intussusception, and intestinal obstruction. ${ }^{1-4}$ Diagnosis with clinical symptoms, hematological investigations, and biochemical profile is usually inconclusive. Testing the stool for ova is one of the initial tests that are done which aid in establishing a diagnosis. Our patient failed to pass stool after he was admitted to the hospital. Abdominal X-rays, ultrasound, and CT scans may be used to rule out other differentials that might give a similar presentation and visualize the $\mathrm{AL}$ itself. ${ }^{5}$ In the case of our patient, following an inconclusive abdominal X-ray and CT scan, barium studies were planned and a barium meal and follow through were scheduled. Barium studies were performed due to the clinical presentation of the patient in which upper intestinal obstruction was more likely the cause of discomfort. An abdominal $\mathrm{X}$-ray and whole abdomen $\mathrm{CT}$ were within reference range, indicating that the large intestine had no abnormalities that could lead to the green vomitus the patient had initially presented with. Barium investigation revealed Ascaris as the culprit of the child's symptoms. Exactly $250 \mathrm{~mL}$ of contrast was given to the patient and a series of images were taken thereafter. The first picture taken was right after the patient had completely ingested the contrast, and the last image was taken 1 hour after its consumption. Barium study 
evaluation was conducted through to the terminal ileum. A low-count AL infestation usually presents as a diagnostic dilemma, as reported by Lakshmi et $\mathrm{al}^{6}$ - as the small group of worms keep migrating to more favorable areas, or the worm burden is very low to begin with. Low worm counts may mimic several diseases, confounding the original diagnosis, hence delaying the treatment to relieve acute intestinal obstruction.

X-ray of the abdomen in intestinal obstruction may show several air fluid levels. Mortality rate of intestinal obstruction of children under the age of 10 years is below $6 \%$. Partial obstruction of the intestines from AL infestation usually resolves spontaneously with conservative treatment which includes bowel rest, intravenous fluids, and nasogastric decompression. ${ }^{7}$ If the mechanical obstruction persists, the worms act as a fixed point leading to intussusception or volvulus formation. ${ }^{1,8}$ AL may excrete toxins, leading to small bowel spasticity and inflammation. Volvulus, intussusception, or increasing pressure to the intestinal wall will inevitably cause intestinal necrosis. If intestinal necrosis occurs, resection and primary anastomosis are a mandatory step in the management of the disease. Medical therapy consists of treatment with antihelminthic drugs, mainly albendazole and mebendazole. ${ }^{7}$ A single dosing is usually curative in $85 \%$ of the patients. Multiple dosing can be administered taking into account the worm count, efficacy, and adverse effects of the drugs. ${ }^{9}$

\section{Conclusion}

AL should be kept in mind in preschool children with symptoms of sudden acute intestinal obstruction. Barium studies are effective, simple, and a cheap alternative to CT abdomen in the diagnosis of acute-onset intestinal obstruction secondary to AL infestation, if other imaging studies turn out inconclusive. The patient should be managed medically if bowel necrosis has not yet occurred. Moderate to severe AL infestation causing partial to almost complete intestinal obstruction managed medically results in a lesser hospital stay and lower mortality rates, in comparison to surgical management. AL infestation is easier to diagnose in cases of a heavy worm count causing intestinal obstruction. Partial bowel obstruction may often present as a confounding mix of several diseases, none with a characteristic diagnostic marker to reach a definitive diagnosis.

\section{Disclosure}

The authors report no conflicts of interest on this work.

\section{References}

1. Mishra PK, Agrawal A, Joshi M, Sanghvi B, Shah H, Parelkar SV. Intestinal obstruction in children due to Ascariasis: A tertiary health centre experience. Afr J Paediatr Surg. 2008;5:65-70.

2. Villamizar E, Méndez M, Bonilla E, Varon H, de Onatra S. Ascaris lumbricoides infestation as a cause of intestinal obstruction in children: experience with 87 cases. J Pediatr Surg. 1996;31:201-205.

3. Spillmann RK. Pulmonary ascariasis in tropical communities. Am JTrop Med Hyg. 1975;24(5):791-800.

4. Varga M, Dumitraşcu D, Piloff L, Chioreanu E. Skin manifestations in parasite infection. Roum Arch Microbiol Immunol. 2001;60(4):359-369.

5. DiSantis DJ, Ralls PW, Balfe DM, et al. The patient with suspected small bowel obstruction: imaging strategies. American College of Radiology. ACR Appropriateness Criteria. Radiology. 2000;215(suppl):121-124.

6. Lakshmi SP, Palaniappan N, Arunagiri A. Ascariasis: challenges in the diagnosis of single worm disease. $J$ Indian Med Assoc. 2013;111(9):621-622.

7. Gangopadhyay AN, Upadhyaya VD, Gupta DK, Sharma SP, Kumar V. Conservative treatment for round worm intestinal obstruction. Indian $J$ Pediatr. 2007;74:1085-1087.

8. Rodriguez EJ, Gama MA, Ornstein SM, Anderson WD. Ascariasis causing small bowel volvulus. Radiographics. 2003;23(5):1291-1293.

9. Adegnika AA, Zinsou JF, Issifou S, et al. Randomized, controlled, assessor-blind clinical trial to assess the efficacy of single- versus repeated-dose albendazole to treat Ascaris lumbricoides, Trichuris trichiura, and hookworm infection. Antimicrob Agents Chemother. 2014;58(5):2535-2540.

\section{Publish your work in this journal}

The International Journal of General Medicine is an international, peer-reviewed open-access journal that focuses on general and internal medicine, pathogenesis, epidemiology, diagnosis, monitoring and treatment protocols. The journal is characterized by the rapid reporting of reviews, original research and clinical studies across all disease areas.

\section{Dovepress}

A key focus is the elucidation of disease processes and management protocols resulting in improved outcomes for the patient. The manuscript management system is completely online and includes a very quick and fair peer-review system. Visit http://www.dovepress.com/ testimonials.php to read real quotes from published authors. 